\title{
ERROR 404 - Sleep not found
}

\section{George L Muntingh' ${ }^{1}$ Michelle Viljoen ${ }^{2}$}

'Tygerberg Hospital Pharmacy, Tygerberg, Western Cape, 7505

2Department of Pharmacology and Clinical Pharmacy, School of Pharmacy, The Faculty of Natural Sciences, University of the Western Cape *Corresponding author, email: george.muntingh@westerncape.gov.za

\begin{abstract}
Insomnia is perhaps the most common type of sleep disorder in the family medicine population. It is best described as a persistent difficulty initiating or maintaining sleep, or a report of non-restorative sleep, accompanied by related daytime impairment. There is increasing evidence of a strong association between insomnia and various medical and psychiatric comorbidities. Diagnosis of insomnia and treatment planning rely on a thorough sleep history to address contributing and precipitating factors as well as maladaptive behaviours resulting in poor sleep. Cognitive behavioural therapy for insomnia (CBT-I) is the mainstay of treatment and is a safe and effective approach. The key challenge of CBT-I is the lack of clinicians to implement it. The newer generation nonbenzodiazepines (e.g. zolpidem) are used as first-line pharmacotherapy for chronic insomnia. Newer drugs active on targets other than the gamma-aminobutyric acid receptor are now available, but clear treatment guidelines are needed.
\end{abstract}

Keywords: insomnia, hypnotic, benzodiazepines, treatment

\section{Introduction}

Oh sleep, Oh gentle sleep, Nature's soft nurse, how have I frightened thee? That thou no more wilt weigh mine eyelids down, And steep my senses in forgetfulness?

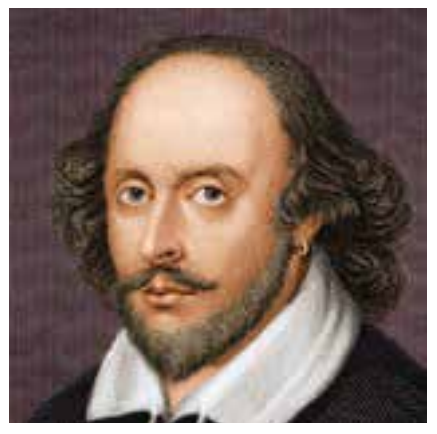

Henry IV, William Shakespeare

Charles Henry Wheeler. The Dramatic Works of W. Shakespeare with Glossarial Notes. A Sketch of His Life, and an estimate of his writings. Published by Fisher, Son \& Co. LONDON, 1830:423

The word "insomnia' originates from the Latin "in" (no) and "somnus" (sleep). Being the first psychosomatic disorder to be described by Johann Heinroth in 1818 (Figure 1), insomnia clinically presents as a subjective perception of dissatisfaction with the amount and/or quality of the sleep. ${ }^{1}$

Insomnia was previously viewed as a sleep disturbance that was secondary to a medical condition, psychiatric illness, sleep disorder, or medication, and would improve with treatment of the underlying disorder. However, evidence over the past 20 years indicates that this view is incorrect. ${ }^{2}$

It is now recognised that insomnia is often an independent disorder. ${ }^{3,4}$ Insomnia may occur in the absence of coexisting conditions and, when coexisting conditions exist, may persist despite successful treatment of the coexisting condition.

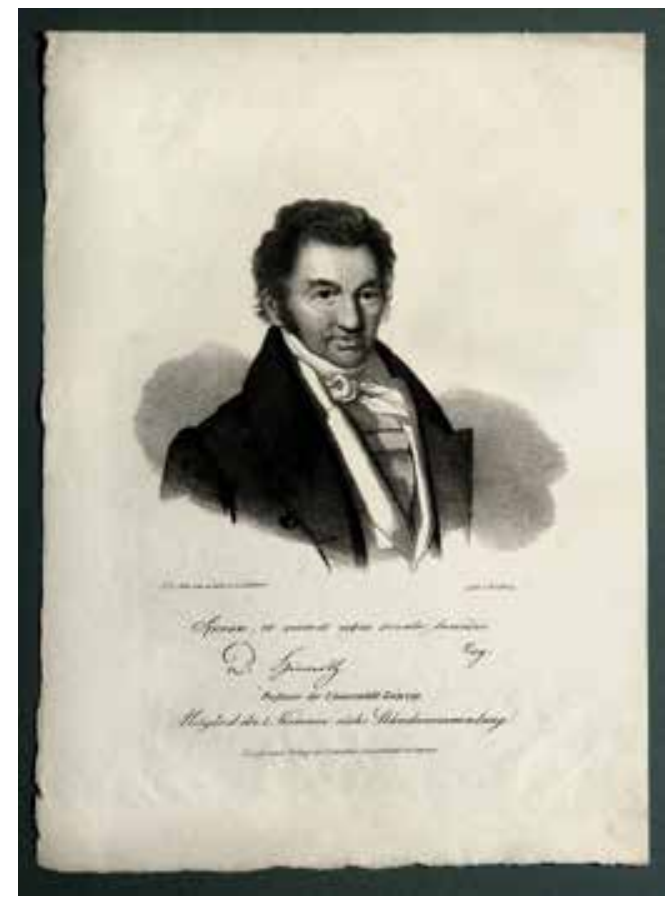

Figure 1. Johann Heinroth

https://upload.wikimedia.org/wikipedia/commons/5/52/Kopie_von_Johann_ Christian_August_Heinroth.JPG - Retrieved 25-05-2018

Treatment directed at the insomnia and the comorbidity may be necessary. Since insomnia can precipitate, exacerbate, or prolong comorbid conditions, treatment of insomnia may improve comorbidities. ${ }^{5-8}$

Patients with insomnia have impaired daytime function due to difficulty initiating sleep, difficulty maintaining sleep, or waking 
up early in the morning without ability to return to sleep. ${ }^{9}$ This occurs despite having adequate time and opportunity for sleep.

Insomnia is present when all three of the following criteria are met $^{10}$ :

- A complaint of difficulty initiating sleep, difficulty maintaining sleep, or waking up too early. In children or individuals with dementia, the sleep disturbance may manifest as resistance to going to bed at the appropriate time or difficulty in sleeping without caregiver assistance.

- The above sleep difficulty occurs despite adequate opportunity and circumstances for sleep.

- The impaired sleep produces deficits in daytime function.

More elaborate criteria is demonstrated by the DSM- 5 criteria for insomnia which includes the following (Table 1) ${ }^{10}$ :

Table 1. The DSM-5 criteria for insomnia

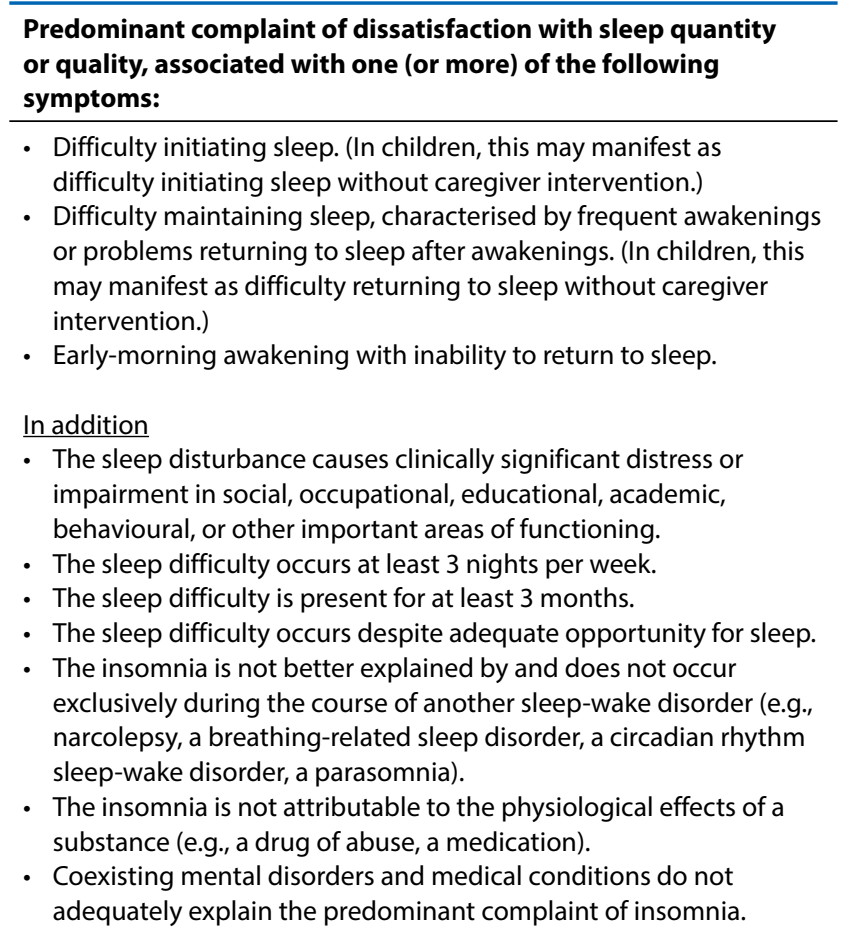

Various studies have noted insomnia to be quite a common condition with symptoms present in about $33-50 \%$ of the adult population. ${ }^{11}$ The prevalence, however, ranges from 10 to $15 \%$ among the general population, ${ }^{12}$ with higher rates seen among divorced, separated, or widowed people, ${ }^{13}$ older ages, female gender, ${ }^{14}$ White population, ${ }^{15}$ and in the presence of co-morbid medical or psychiatric illness. ${ }^{16}$ About $30 \%$ of all adults complain of occasional insomnia and $10 \%$ of chronic insomnia, of whom $40 \%$ may have a psychiatric illness. ${ }^{17,18}$ Despite these high prevalence rates, evidence suggests that insomnia is mostly under-recognised, under-diagnosed, and under-treated, with the condition continuing to remain persistent in $50-85 \%$ of individuals over follow-up intervals of one to several years. ${ }^{19}$

Chronic insomnia represents a more complex condition than acute transient insomnia. Patients with chronic insomnia usually have accompanying daytime impairment of cognition, mood, or performance that impacts not only the patient and his family, but also affects friends, coworkers, and caretakers. Insomnia patients are more likely to visit hospitals and physicians, have increased absenteeism, make errors or have accidents at work, and have more fatal road accidents. ${ }^{20,21}$ There is also an increased risk for depression, anxiety, substance use, suicide, and possible immune dysfunction. ${ }^{22}$ It is therefore imperative that clinicians remain alert to these possible individual and societal risks during the evaluation.

\section{Therapy for insomnia - general approach}

"The best cure for insomnia is to get a lot of sleep." WC Fields ${ }^{73}$

All patients with insomnia should receive therapy for any medical condition, psychiatric illness, substance abuse, or sleep disorder that may be precipitating or exacerbating the insomnia. They should also receive basic behavioural counselling about sleep hygiene and stimulus control.

For patients who continue to have insomnia that is sufficiently burdensome to warrant other interventions, treatment options include behavioural therapy, medication, or both (Table 2):

Table 2. Treatment options for insomnia ${ }^{23,24}$

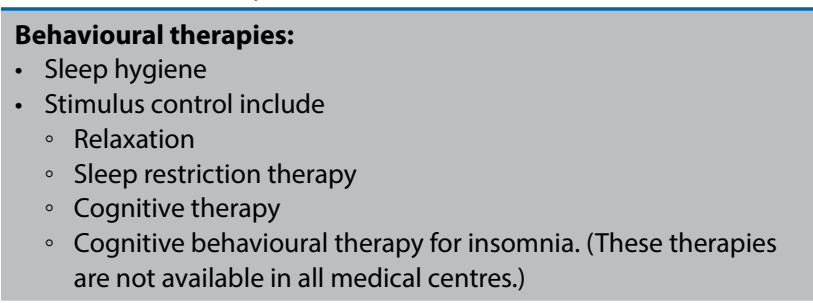

Pharmacotherapy options to treat insomnia include:

- Benzodiazepines

- Nonbenzodiazepine sedatives

- Melatonin agonists

- Doxepin

- Suvorexant (an orexin antagonist).

Combination therapy involves:

- Initially prescribing both CBT-I and a medication (usually for six to eight weeks), then

- Tapering the medication off or to an as-needed schedule while continuing CBT-I. The use of medication prior to the initiation of behavioural therapy appears to be less effective.

The choice of treatment should be individualised according to the patient's values and preferences, the availability of advanced behavioural therapies, the severity and impact of the insomnia, and the potential benefits versus the risks, costs, and inconveniences. For most patients, it is suggested to engage CBT-I rather than medication as initial therapy, recognising that both approaches are effective in short-term studies, but that medications have a higher risk of side effects and have been inadequately evaluated for use for longer than a year. However, access to behavioural therapy may be limited in some regions and for some patient groups. A preference for CBT-I or other behavioural therapies over medication as initial therapy has been endorsed in clinical practice guidelines of the American Academy of Sleep Medicine, ${ }^{25}$ the British Association for Psychopharmacology ${ }_{1}^{26}$ the American College of Physicians, ${ }^{27}$ and the European Sleep Research Society. ${ }^{28}$ 
Treatment decisions must also factor in the potential health risks of untreated chronic insomnia, which include decreased quality of life, increased risk for psychiatric comorbidities and substance abuse, decreased performance, and the association between chronic insomnia and risk of cardiovascular morbidity and allcause mortality.

In clinical practice, initial treatment typically involves sleep hygiene instruction and stimulus control procedures. If followup indicates that further treatment is needed, then more formal CBT-I alone or in combination with a medication may be used for six weeks. For patients who respond to therapy (i.e., report both improved sleep at night and improvement of daytime deficits), the medication can be tapered or used as needed while continuing the CBT-I. Patients whose symptoms recur after discontinuation of therapy may require re-evaluation for referral for polysomnography or additional CBT-I, with or without pharmacologic therapy.

Perhaps an exception to this approach is patients who have short-term insomnia due to a self-limited stressor; such patients may benefit from short-term medication alone. ${ }^{28}$

\section{Pharmacotherapy Options}

Pharmacological interventions for insomnia include different classes of medications, with differing mechanisms of action, reflecting the multiple neural systems that regulate sleep.

Medications or classes of medications that are approved and available to treat insomnia include benzodiazepines, nonbenzodiazepine hypnotics, melatonin agonists, doxepin, and suvorexant (Table 3). ${ }^{29,30}$

Although all of these agents or classes have been shown to be more effective than placebo at improving short-term sleep outcomes, the magnitude of effect is variable. The potential benefits of pharmacologic therapy on sleep quality and daytime function are balanced against the risk of side effects as well as physical and psychological addiction with long-term use. Risks that should be considered and which may increase side-effects in certain clinical settings include: ${ }^{30}$

- Pregnancy - Sedative-hypnotics may increase the risk of fetal malformations if used during the first trimester.

- Alcohol consumption - Sedative-hypnotics should not be combined with alcohol because there is a risk of excessive sedation and respiratory suppression whenever central nervous system suppressants are combined.

- Renal or hepatic disease - Most sedative-hypnotic medications undergo hepatic and renal clearance. Metabolic clearance may be delayed in patients who have renal or hepatic disease, leading to accumulation and excessive sedation.

- Pulmonary disease or sleep apnoea - Many sedative-hypnotics can worsen obstructive sleep apnoea or hypoventilation.

- Night time decision makers - Sedative-hypnotics should not be taken by individuals who may be called upon to make important decisions during the night (e.g., clinicians on-call or single parents responsible for the care of young children) because they can cause excess sedation and of course anterograde amnesia.

- Older adults - The risk of adverse effects is increased in older adults, especially those who are older than 75 years. This is a consequence of multiple comorbidities and central nervous system changes associated with aging.

\section{Hypnotics}

The modern history of sedative hypnotics began with barbital, which was introduced in 1903, and later phenobarbital in 1912. In the mid-1930s, Sternbach synthesised several heptoxdiazines, although it was not until 1955 when one of these quinazolines was treated with methylamine that an active compound was developed. In 1957, the compound chlordiazepoxide was found to have hypnotic, sedative, and muscle-relaxing effects. The benzodiazepines provided advantages over the older barbiturates because they were less toxic in overdose and had fewer drug interactions. In addition, they had superior efficacy and safety compared to meprobamate, which was introduced as a tranquiliser in 1955. Non-benzodiazepine hypnotics have been the most recent addition and may offer some advantages of lower risk tolerance and perhaps lower abuse liability, although clinical experience is insufficient to make definitive judgments. ${ }^{31}$

Hypnotic (from Greek hypnos, sleep) or also called a somnifacient (an agent that causes sleep) drugs, commonly known as sleeping pills, are a class of psychoactive drugs whose primary function is to induce sleep and to be used in the treatment of insomnia (sleeplessness), or surgical anesthesia. ${ }^{32}$

This group is related to sedatives. Whereas the term sedative describes drugs that serve to calm or relieve anxiety, the term hypnotic generally describes drugs whose main purpose is to initiate, sustain, or lengthen sleep. Because these two functions frequently overlap, and because drugs in this class generally produce dose-dependent effects (ranging from anxiolysis to loss of consciousness) they are often referred to collectively as sedative-hypnotic drugs. ${ }^{33}$

\section{Benzodiazepines}

Benzodiazepines bind to the unique benzodiazepine receptor binding site of the GABAA receptor complex, resulting in agonistic effects on GABA-ergic transmission and hyperpolarisation of neuronal membranes. The increase in intrinsic activity of the inhibitory neurotransmitter GABA enhances inhibitory outputs to all the major cell groups in the brainstem and hypothalamus that promote arousal. Benzodiazepines enhance the sleeppromoting effects of homeostatic sleep-drive and decrease activity in the arousal systems (e.g., histamine, serotonin, acetylcholine and orexin). They therefore also relieve insomnia by reducing sleep latency and increasing total sleep time. ${ }^{34-37}$

Benzodiazepines commonly used for the treatment of insomnia include triazolam, estazolam, lorazepam, temazepam, flurazepam, and quazepam. A primary difference among these medications is their duration of action. 
Triazolam is short acting; estazolam, lorazepam, and temazepam are intermediate acting; flurazepam and quazepam are long acting. ${ }^{38}$ The long-acting benzodiazepines should be avoided in older adults because there is increased risk for adverse effects in this patient population. ${ }^{39}$

Meta-analyses of randomised, placebo-controlled trials indicate that benzodiazepines decrease sleep latency and the number of awakenings, while improving sleep duration and sleep quality..$^{40-43}$

Typical changes associated with these medications include decreases in the duration to sleep onset by approximately 10 minutes and increases in the total sleep time of 30 to 60 minutes. ${ }^{40,41}$

\section{Nonbenzodiazepines}

In the 1980s and early 1990s, a new group of hypnotic agents, known as new-generation hypnotics, nonbenzodiazepine hypnotics, benzodiazepine receptor agonists or 'z-drugs' were introduced to the markets. ${ }^{44}$

Nonbenzodiazepine benzodiazepine receptor agonists have a structure that is different from the benzodiazepines and include more targeted action at one kind of GABA type A receptor. A consequence of their greater specificity is less anxiolytic and anticonvulsant activity.

Nonbenzodiazepines appear to improve both subjective and objective sleep outcomes. Specifically, meta-analyses of randomised, placebo-controlled trials indicate that nonbenzodiazepines decrease sleep latency and the number of awakenings, while improving sleep duration and sleep quality. ${ }^{41,42} 44,45$

Nonbenzodiazepines commonly used to treat insomnia include zaleplon, zopiclone, eszopiclone (not available in South Africa yet), zolpidem, and zolpidem extended release:

1. Zaleplon has a very short half-life of about one hour. As a result, it is effective for patients who have difficulty falling asleep (i.e., sleep onset insomnia), but may not be effective for patients who have difficulty maintaining sleep (i.e., sleep maintenance insomnia). ${ }^{47}$ Due to the very short half-life, the potential for hangover sleepiness is minimal after normal sleep periods. Occasional side effects include headache, dizziness, nausea, abdominal pain, and somnolence. ${ }^{46}$ Zaleplon is not indicated for long-term use.

2. Zolpidem has a half-life of approximately 1.4 to 4.5 hours. It is indicated for the short-term treatment of insomnia characterised by difficulty with sleep initiation. The most common side effects are headache, dizziness, and somnolence, which can in turn lead to falls. Zolpidem is not approved for long-term use. ${ }^{48}$

Zolpidem extended release has a half-life of about 1.4 to 4 hours, but is released over a longer duration. It was developed to improve both sleep onset insomnia and sleep maintenance insomnia while avoiding hangover effects, although it has never been directly compared with regular zolpidem. ${ }^{49}$
Side effects of zolpidem extended release are relatively few, with the most common being headache, somnolence, and dizziness, which can in turn lead to falls (especially in the elderly patient). This lower dose should also be considered for men. In a follow-up safety announcement, the FDA added a warning that patients should not drive or engage in other activities that require complete mental alertness the day after taking zolpidem extended release because zolpidem levels can remain high enough the next day to impair these activities. $^{48,50}$

Sleep may be worse during the first night following discontinuation of this medicine. Zolpidem extended release is not limited to short-term use and there is little evidence for abuse or dependence in most patients. In theory, however, such medications could be habit forming with long-term use.

3. Zopiclone is approved with doses of $7.5 \mathrm{mg}$ for adults and $3.75 \mathrm{mg}$ for elderly patients. It is rapidly absorbed (maximum plasma concentration (Tmax) 1.5 to 2 hours) and has a relatively long elimination half-life time $\left(t \frac{1}{2}\right) \approx 5$ to 6 hours) compared to other new-generation hypnotic compounds. Metabolites of zopiclone are assumed to have similar elimination half-lives to that of the parent compound, whereby the active zopiclone metabolite (S)-desmethylzopiclone was shown to be present eight hours after zopiclone dosing. ${ }^{44}$

4. Eszopiclone has the longest half-life of the approved nonbenzodiazepines, approximately six hours. This may extend to nine hours in older adult patients. Eszopiclone is effective for both sleep onset insomnia and sleep maintenance insomnia. ${ }^{51}$ Patients taking eszopiclone may report an unpleasant metallic taste. Other reported side effects are shared with nonbenzodiazepines as a class (headache, dizziness, parasomnias, next-day impairment in some patients). ${ }^{52}$

Sleep may be worse on the first night after discontinuation of this medication. Eszopiclone is not limited to short-term use and there is little evidence for abuse or dependence in most patients. In theory, however, such medications could be habit forming with long-term use.

Most clinical trials evaluated short-term therapy (e.g. seven days), although a few longer clinical trials have been performed. In one randomised trial, patients treated with eszopiclone for six months had improved quality of life, decreased work limitation, and improved sleep compared with placebo. This persisted throughout the trial and the subsequent six-month open label extension. In another randomised trial of 1018 patients with insomnia, zolpidem extended release taken for up to six months improved sleep onset, sleep maintenance, morning sleepiness, nextday concentration, and work performance compared with placebo. $^{53,54}$ 
Table 3. Summary of hypnotics for insomnia 55

\begin{tabular}{|c|c|c|c|c|c|}
\hline Drug Class & Generic Name & Dose (mg) & Eliminations Half Life (h) & $\operatorname{Tmax}(\mathrm{h})$ & Indication \\
\hline \multirow{5}{*}{ Benzodiazepines } & Estrazolam & 1,2 & $10-24$ & $1.5-2$ & * SOI, SMI \\
\hline & Flurazepam & 15,30 & $48-120$ & $1.5-4.5$ & SOI, SMI \\
\hline & Quazepam & $7.5,15$ & $48-120$ & $2-3$ & SOI, SMI \\
\hline & Temazepam & $7.5,15$ & $8-22$ & $1-2$ & SOI, SMI \\
\hline & Triazolam & $0.125,0.25$ & $2-4$ & $2-6$ & SOI \\
\hline \multirow{5}{*}{ Benzodiazepine Agonists } & Zaleplon & $5,10,20$ & 1 & 1 & SOI \\
\hline & Zolpidem & 5,10 & 2.5 & 1.6 & SOI \\
\hline & Zolpidem CR & $6.25,12.5$ & 2.8 & 1.5 & SOI, SMI \\
\hline & Zopiclone & 7.5 & $5-6$ & $1.5-2$ & SOI, SMI \\
\hline & Eszopiclone & $1,2,3$ & 6 & 1 & SOI, SMI \\
\hline Melatonin Agonist & Ramelteon & 8 & $1-2.6$ & 0.75 & SOI \\
\hline Histamine Antagonist & Doxepin & 3,6 & 15.3 & 3.5 & SMI \\
\hline Orexin Antagonist & Suvorexant & $5-20$ & 12 & 2 & SOI, SMI \\
\hline
\end{tabular}

* SOI (Sleep Onset Insomnia); SMI (Sleep Maintenance Insomnia)

\section{Antidepressants (sedating antidepressants)}

These are alternatives to benzodiazepines, especially for patients who have depression or are at risk of substance abuse. The sedating antidepressants, such as amitriptyline and doxepin are effective for inducing sleep continuity, taking advantage of the antihistaminergic, anticholinergic and serotonergic and adrenergic antagonistic activity of these agents. Doxepin, a tricyclic antidepressant, is approved for the treatment of insomnia at dosages of 3-6 mg and has shown significant effects on sleep maintenance, but no benefit for sleep-onset latency beyond two days of treatment. ${ }^{56}$ Mirtazepine is another sedating antidepressant that may help patients sleep, but also causes daytime sedation and weight gain. Trazodone and amitriptyline can also improve sleep continuity and is popular for treating insomnia in patients prone to substance abuse and bupropioneinduced insomnia, as these agents do not cause dependence. Other side-effects that might be experienced include morning sedation, orthostatic hypotension (at higher doses) and priapism. Trazodone and amitriptyline has also shown efficacy for insomnia co-occurring with major depression. ${ }^{57}$

Few data support the use of these agents, although they have been increasingly used. These drugs are associated with substantial side-effects, such as rebound insomnia after withdrawal, arrhythmias and liver dysfunction. They are not approved for long-term use and may require medical monitoring; however, the major advantage of these agents is the absence of dependency. ${ }^{58}$

\section{Miscellaneous Agents (Antihistamines)}

The off-label use of sedating antidepressants and the use of antihistamines are common for insomnia management. Antihistamines, such as diphenhydramine and doxylamine (available as OTC-medications) are generally safe and effective for treating mild insomnia. Patients, however, quickly experience tolerance to the sedative effects and the anticholinergic sideeffects such as constipation, dry mouth, ataxia, urinary retention and extended drowsiness, especially in the elderly. ${ }^{57}$

A summary of some of the medications is given in Table 4.

\section{Some newer pharmacological interventions}

\section{Melatonin}

Melatonin - Melatonin is a hormone that is normally secreted by the pineal gland. It is not recommended as a treatment for insomnia in most patients, ${ }^{59}$ except when sleep disturbances are due to delayed sleep-wake phase syndrome (a circadian sleepwake rhythm disorder). It appears to be somewhat effective and also safe when used short-term (three months or less). ${ }^{60,61}$

\section{Melatonin Receptor Agonist (Ramelteon)}

Ramelteon is a melatonin receptor agonist and FDA- approved for treatment of sleep-onset insomnia. ${ }^{62}$ It is selective for the MT1 and MT2 melatonin receptors and thought to regulate the circadian rhythm and sleep-onset. Ramelteon is associated with reduced subjective sleep latency and improved sleep quality efficiency. ${ }^{63}$ It is proven to effectively treat sleep-onset

Table 4. Summary of Off-label medications for insomnia ${ }^{55}$

\begin{tabular}{|c|c|c|c|c|}
\hline Drug Class & Generic Name & Dose (mg) & Eliminations Half Life (h) & $\operatorname{Tmax}(\mathrm{h})$ \\
\hline \multirow{3}{*}{ Sedating Antidepressants } & Trazodone & $25-150$ & $9(7-15)$ & $1-2$ \\
\hline & Amitriptyline & $10-100$ & $30(5-45)$ & $2-5$ \\
\hline & Doxepin & 3,6 & 15.3 & 3.5 \\
\hline Sedating Antipsychotics & Quetiapine & $25-100$ & 6 & $1-2$ \\
\hline Anihistamines & Diphenhydramine & $25-50$ & $4-8$ & $1-4$ \\
\hline Anticonvusants & Gabapentin & $100-900$ & $5-9$ & $1.6-3$ \\
\hline
\end{tabular}


difficulties in patients with sleep-apnoea and chronic obstructive pulmonary disease. The most common adverse events are somnolence, headache, an unpleasant taste and dizziness; however, ramelteon is generally well-tolerated..$^{64,65}$

\section{Orexin Receptor Antagonist (Suvorexant)}

The novel pharmacological agent suvorexant, an orexin receptor antagonist, was approved by the FDA in 2014 for treatment of insomnia disorder. Orexin is a peptide produced by thousands of neurons located in the posterior lateral hypothalamus.

These neurons project to the same targets that GABA inhibits and orexin thus leads to diminished activity in sleep-promoting areas, enhancing wakefulness, as well as being involved in the stabilisation of the sleep-wake cycle. Suvorexant acts on this orexin system by suppressing the wake-stimulatory network of sleep-wake regulation, leading to decreased time to sleep onset, decreased time awake after sleep onset and increased total sleep time. This is in contrast to the traditional benzodiazepine pharmacological approach, which is to strengthen the sleep drive by augmentation of GABA-ergic activity. Important risks, such as the risk of dependency and tolerance might thus be avoided.66-69 The recommended suvorexant dosage range is 10-20 mg, due to concerns about dose-related, next-day adverse effects, like sedation and cognitive and behavioural changes, such as amnesia and anxiety. ${ }^{70}$

\section{Cannabanoids}

Preliminary research into cannabis and insomnia suggests that cannabidiol (CBD) may have therapeutic potential for the treatment of insomnia. Delta-9 tetrahydrocannabinol (THC) may decrease sleep latency but could impair sleep quality longterm. Novel studies investigating cannabinoids and obstructive sleep apnoea suggest that synthetic cannabinoids such as nabilone and dronabinol may have short-term benefit for sleep apnoea due to their modulatory effects on serotonin-mediated apnoeas. CBD may hold promise for REM sleep behaviour disorder and excessive daytime sleepiness, while nabilone may reduce nightmares associated with PTSD and may improve sleep among patients with chronic pain. Research on cannabis and sleep is in its infancy and has yielded mixed results. Additional controlled and longitudinal research is critical to advance our understanding of research and clinical implications. ${ }^{74}$

\section{Herbal products}

A variety of herbal products are purported to be useful for insomnia. There is little evidence from randomised controlled trials regarding the efficacy of many herbals, however, and for those that have been well studied (e.g., valerian), there is little evidence of benefit. A meta-analysis that included 14 randomised trials in over 1600 patients found no significant difference between any herbal medicine and placebo on any of 13 clinical efficacy measures of insomnia. ${ }^{71}$ The majority of the trials ( 11 out of 14) studied valerian; chamomile, kava, and wuling were studied in one trial each. Unlike the other herbals studied, valerian was associated with a greater number of adverse events per person compared with placebo. Valerian may also produce hepatotoxic effects. ${ }^{72}$ Contamination with undesirable substances poses a problem for many such natural remedies.

\section{Conclusion}

Increased awareness for effective insomnia disorder treatment is fundamentally important. The goal of treatment for insomnia is to improve sleep and alleviate anxiety or dysfunction caused by the disorder. Management of insomnia in all patients should include identifying the cause of insomnia and possible coexisting disorders. Insomnia can be managed with psychological therapy, which includes CBT, pharmacological therapy or a combination of both. Deciding whether to use pharmacological or behavioural therapy is difficult, as both have their limitations and advantages. Research has shown that CBT, which is noninvasive was effective in treating the general population of adults, as well as elderly patients with chronic insomnia disorder. Furthermore, CBT is non-invasive and has been shown to likely have fewer harms, such as addiction to pharmacological agents. Medications, however, usually produce rapid improvements and are widely available, but the risk of adverse-effects (e.g., daytime sedation) and risk of dependence may complicate their use.

\section{References}

1. Mellinger GD, Balter MB, Uhlenhuth EH. Insomnia and its treatment. Prevalence and correlates. Arch Gen Psychiatry. 1985;42:225-32.

2. National Institutes of Health. National Institutes of Health State of the Science Conference statement on Manifestations and Management of Chronic Insomnia in Adults, 13-15 June 2005;28:1049.

3. Katz DA, McHorney CA. Clinical correlates of insomnia in patients with chronic illness. Arch Intern Med. 1998; 158:1099.

4. Krystal AD. Psychiatric comorbidity: the case for treating insomnia. Sleep Med Clin. 2006;1:359.

5. Fava M, McCall WV, Krystal A, et al. Eszopiclone co-administered with fluoxetine in patients with insomnia coexisting with major depressive disorder. Biol Psychiatry. 2006; 59:1052.

6. Roehrs T, Hyde M, Blaisdell B, et al. Sleep loss and REM sleep loss are hyperalgesic. Sleep. 2006;29:145.

7. Tashjian RZ, Banerjee R, Bradley MP, et al. Zolpidem reduces postoperative pain fatigue, and narcotic consumption following knee arthroscopy: a prospective randomized placebo-controlled double-blinded study. Journal of Knee Surgery. 2006;19:105.

8. Sack RL, Auckley D, Auger RR, et al. Circadian rhythm sleep disorders: part II, advanced sleep phase disorder, delayed sleep phase disorder, free-running disorder, and irregular sleep-wake rhythm. An American Academy of Sleep Medicine review. Sleep. 2007;30:1484

9. American Academy of Sleep Medicine. International Classification of Sleep Disorders, 3rd ed, American Academy of Sleep Medicine, Darien, IL 2014.

10. "Sleep Wake Disorders" Diagnostic and statistical manual of mental disorders: DSM-5. 5th ed. Washington, DC: American Psychiatric Association, 2013.

11. Ancoli-Israel S, Roth T. Characteristics of insomnia in the United States: Results of the 1991 National Sleep Foundation Survey. I. Sleep. 1999;22:S347-53.

12. Roth T. New developments for treating sleep disorders. J Clin Psychiatry. 2001;62:3-4.

13. Dollander M. Etiology of adult insomnia. Encephale. 2002;28:493-502.

14. Hohagen F, Rink K, Käppler C, Schramm E, Rieman D, Weyerer S, et al. Prevalence and treatment of insomnia in general practice: A longitudinal study. Eur Arch Psych Clin Neurosc. 1993;242:325-36.

15. Riedel BW, Durrence HH, Lichstein KL, Taylor DJ, Bush AJ. The relation between smoking and sleep: The influence of smoking level, health, and psychological variables. Behav Sleep Med. 2004;2:63-78.

16. Buscemi N, Vandermeer B, Friesen C, Bialy L, Tubman M, Ospina M, et al. Manifestations and management of chronic insomnia in adults. Evidence Report/Technology Assessment (Summ) 2005;125:1-10.

17. Benca RM. Diagnosis and treatment of chronic insomnia: A review. Psychiatric Services. 2005;56:332-43.

18. Franzen PL, Buysse DJ. Sleep disturbances and depression: Risk relationships for subsequent depression and therapeutic implications. Dialogues Clin Neurosci. 2008;10:473-81.

19. Morin CM, LeBlanc M, Daley M, Gregoire JP, Merette C. Epidemiology of insomnia: Prevalence, self-help treatments, consultations, and determinants of help-seeking behaviors. Sleep Med. 2006;7:123-30.

20. Leger D, Guilleminault C, Bader G, Levy E, Paillard M. Medical and socio-professional impact of insomnia. Sleep. 2002;25:625-9. 
21. Léger D, Massuel MA, Metlaine A. SISYPHE Study Group. Professional correlates of insomnia. Sleep. 2006;29:171-8.

22. Taylor DJ, Lichstein $\mathrm{KL}$, Durrence $\mathrm{HH}$. Insomnia as a health risk factor. Behav Sleep Med. 2003;1:227-47.

23. Brasure M, Fuchs E, MacDonald R, et al. Psychological and Behavioral Interventions for Managing Insomnia Disorder: An Evidence Report for a Clinical Practice Guideline by the American College of Physicians. Ann Intern Med. 2016;165:113.

24. Vallières $A$, Morin CM, Guay B. Sequential combinations of drug and cognitive behavioral therapy for chronic insomnia: an exploratory study. Behav Res Ther. 2005;43:1611.25.

25. Schutte-Rodin S, Broch L, Buysse D, et al. Clinical guideline for the evaluation and management of chronic insomnia in adults. J Clin Sleep Med. 2008;4:487.26.

26. Wilson SJ, Nutt DJ, Alford C, et al. British Association for Psychopharmacology consensus statement on evidence-based treatment of insomnia, parasomnias and circadian rhythm disorders. J Psychopharmacol. 2010;24:1577.

27. Qaseem A, Kansagara D, Forciea MA, et al. Management of Chronic Insomnia Disorder in Adults: A Clinical Practice Guideline From the American College of Physicians. Ann Intern Med. 2016;165:125.

28. Riemann D, Baglioni C, Bassetti C, et al. European guideline for the diagnosis and treatment of insomnia. J Sleep Res. 2017;26:675.

29. Wilt TJ, MacDonald R, Brasure M, et al. Pharmacologic Treatment of Insomnia Disorder: An Evidence Report for a Clinical Practice Guideline by the American College of Physicians. Ann Intern Med. 2016;165:103.

30. Sateia MJ, Buysse DJ, Krystal AD, et al. Clinical Practice Guideline for the Pharmacologic Treatment of Chronic Insomnia in Adults: An American Academy of Sleep Medicine Clinical Practice Guideline. J Clin Sleep Med. 2017;13:307.

31. Ciraulo DA, Oldham M. 16: Sedative Hypnotics. The Effects of Drug Abuse on the Human Nervous System. Academic Press. 2014; 499-532.

32. Definition of hypnotic. Accessed on 23 May 2018. Available from: https:// medical-dictionary.thefreedictionary.com/somnifacient.

33. Brunton L, Lazo JS, Parker KL. "17: Hypnotics and Sedatives". Goodman \& Gilman's The Pharmacological Basis of Therapeutics (11th ed). The McGraw-Hill Companies, Inc. 2006 (Accessed on 6 Feb 2018). ISBN 0-07-146804-8.

34. Morin CM. Definition of acute insomnia: diagnostic and treatment implications. Sleep Med Rev. Feb 2012;16(1):3-4.

35. Riemann D, Spiegelhalder K, Feige B, Voderholzer U, Berger M, Perlis M, et al. The hyperarousal model of insomnia: a review of the concept and its evidence. Sleep Med Rev. 2010;14(1):19-31.

36. American Academy of Sleep Medicine. International Classification of Sleep Disorders: Diagnostic and Coding Manual. 3rd ed. Westchester, III: American Academy of Sleep Medicine; 2014.

37. Morin CM, Edinger JD, Krystal AD, Buysse DJ, Beaulieu-Bonneau S, Ivers $H$. Sequential psychological and pharmacological therapies for comorbid and primary insomnia: study protocol for a randomized controlled trial. Trials. 2016;17(1):118.

38. Drugs for insomnia - Treat Guidelines. Medical Letter. 2009;7:23.

39. Bloom HG, Ahmed I, Alessi CA, et al. Evidence-based recommendations for the assessment and management of sleep disorders in older persons. J Am Geriatr Soc. 2009;57:761.

40. Buscemi N, Vandermeer B, Friesen C, et al. The efficacy and safety of drug treatments for chronic insomnia in adults: a meta-analysis of RCTs. J Gen Intern Med. 2007;22:1335.

41. Holbrook AM, Crowther R, Lotter A, et al. Meta-analysis of benzodiazepine use in the treatment of insomnia. CMAJ. 2000;162:225.

42. Nowell PD, Mazumdar S, Buysse DJ, et al. Benzodiazepines and zolpidem for chronic insomnia: a meta-analysis of treatment efficacy. JAMA. 1997;278:2170.

43. Krystal AD. A compendium of placebo-controlled trials of the risks/benefits of pharmacological treatments for insomnia: the empirical basis for U.S. clinical practice. Sleep Med Rev. 2009;13:265.

44. Nutt DJ, Stahl SM. Searching for perfect sleep: the continuing evolution of GABAA receptor modulators as hypnotics. J Psychopharmacol. 2010;24(11):1601-12.

45. Huedo-Medina TB, Kirsch I, Middlemass $J$, et al. Effectiveness of non-benzodiazepine hypnotics in treatment of adult insomnia: meta-analysis of data submitted to the Food and Drug Administration. BMJ. 2012;345:e8343.

46. Lieberman JA. Update on the safety considerations in the management of insomnia with hypnotics: incorporating modified-release formulations into primary care. Prim Care Companion. J Clin Psychiatry. 2007;9:25.

47. Elie R, Rüther E, Farr I, et al. Sleep latency is shortened during 4 weeks of treatment with zaleplon, a novel nonbenzodiazepine hypnotic. Zaleplon Clinical Study Group. J Clin Psychiatry. 1999;60:536.

48. Risk of next morning impairment after use of insomnia drugs; FDA requires lower recommended doses for certain drugs containing zolpidem (Ambien, Ambien CR, Edluar, and Zolpimist). Safety Announcement. 1 Oct 2013 (Accessed on 1 May 2018). Available from: https://www.fda.gov/downloads/Drugs/ DrugSafety/UCM335007.pdf
49. Hindmarch I, Legangneux E, Stanley N, et al. A double-blind, placebo-controlled investigation of the residual psychomotor and cognitive effects of zolpidem-MR in healthy elderly volunteers. Br J Clin Pharmacol. 2006;62:538.

50. FDA Drug Safety Communication: FDA approves new label changes and dosing for zolpidem products and a recommendation to avoid driving the day after using Ambien CR. Safety Announcement, 14 May 2013 (Accessed on 1 May 2018). Available from: https://www.fda.gov/drugs/drugsafety/ucm352085.htm

51. Roth T, Walsh JK, Krystal A, et al. An evaluation of the efficacy and safety of eszopiclone over 12 months in patients with chronic primary insomnia. Sleep Med. 2005;6:487.

52. Ancoli-Israel S, Krystal AD, McCall WV, et al. A 12-week, randomized, doubleblind, placebo-controlled study evaluating the effect of eszopiclone $2 \mathrm{mg}$ on sleep/wake function in older adults with primary and comorbid insomnia. Sleep. 2010;33:225.

53. Krystal AD, Erman M, Zammit GK, et al. Long-term efficacy and safety of zolpidem extended-release $12.5 \mathrm{mg}$, administered 3 to 7 nights per week for 24 weeks, in patients with chronic primary insomnia: a 6-month, randomized double-blind, placebo-controlled, parallel-group, multicenter study. Sleep. 2008;31:79.

54. Erman M, Guiraud A, Joish VN, Lerner D. Zolpidem extended-release $12.5 \mathrm{mg}$ associated with improvements in work performance in a 6-month randomized, placebo-controlled trial. Sleep. 2008;31:1371.

55. Asnis GM, Thomas M, Henderson MA. Pharmacotherapy Treatment Options for Insomnia: A Primer for Clinicians. Int J Mol. Sci. 2016;17(1):50. doi:10.3390/ ijms17010050

56. Krystal AD, Lankford A, Durrence $H H$, Ludington $E$, Jochelson $P$, Rogowski $R$, Roth T. Efficacy and safety of doxepin 3 and $6 \mathrm{mg}$ in a 35-day sleep laboratory trial in adults with chronic primary insomnia. Sleep. 2011;34(10):1433.

57. Lippmann S, Mazour I, Shahab H. Insomnia: therapeutic approach. South Med J. 2001;94(9):866.

58. Bertisch SM, Herzig SJ, Winkelman JW, Buettner C. National use of prescription medications for insomnia: NHANES 1999-2010. Sleep. 2014;37(2):343-9.

59. Sateia MJ, Buysse DJ, Krystal AD, et al. Clinical Practice Guideline for the Pharmacologic Treatment of Chronic Insomnia in Adults: An American Academy of Sleep Medicine Clinical Practice Guideline. J Clin Sleep Med. 2017;13:307.

60. Buscemi N, Vandermeer B, Hooton N, et al. The efficacy and safety of exogenous melatonin for primary sleep disorders. A meta-analysis. J Gen Intern Med. 2005;20:1151.

61. van Geijlswijk IM, Korzilius HP, Smits MG. The use of exogenous melatonin in delayed sleep phase disorder: a meta-analysis. Sleep. 2010; 33:1605.

62. Buscemi N, Vandermeer B, Friesen C, Bialy L, Tubman M, Ospina M, et al. The efficacy and safety of drug treatments for chronic insomnia in adults: a meta-analysis of RCTs. J Gen Intern Med. 2007;22(9):1335.

63. Belsomra, suvorexant [Product Information]. Whitehouse Station, NJ: Merck and Co., Inc.; 2014.

64. Kuriyama A, Honda M, Hayashino $Y$. Ramelteon for the treatment of insomnia in adults: a systematic review and meta-analysis. Sleep Med. 2014;15(4):385-92.

65. Ferracioli-Oda E, Qawasmi A, Bloch MH. Meta-analysis: melatonin for the treatment of primary sleep disorders. PloS one. 2013;8(5):e63773.

66. Kryger M, Roth $\mathrm{T}$, Wang-Weigand $\mathrm{S}$, Zhang J. The effects of ramelteon on respiration during sleep in subjects with moderate to severe chronic obstructive pulmonary disease. Sleep Breath. 2009;13(1):79-84.

67. Citrome L. Suvorexant for insomnia: a systematic review of the efficacy and safety profile for this newly approved hypnotic-what is the number needed to treat, number needed to harm and likelihood to be helped or harmed? Int J Clin Pract. 2014;68(12):1429-41.

68. Zeitzer JM, Nishino S, Mignot E. The neurobiology of hypocretins (orexins), narcolepsy and related therapeutic interventions. Trends Pharmacol Sci. 2006;27:368-74.

69. Herring WJ, Connor KM, Ivgy-May N, Snyder E, Liu K, Snavely DB, et al. Suvorexant in patients with insomnia: results from two 3-month randomized controlled clinical trials. Biol Psychiatry. 2016;79(2):136-48.

70. Broese M, Riemann D, Hein L, Nissen C. a-Adrenergic receptor function, arousal and sleep: mechanisms and therapeutic implications. Pharmacopsychiatry. 2012;45(06):209-16.

71. Leach MJ, Page AT. Herbal medicine for insomnia: A systematic review and meta-analysis. Sleep Med Rev. 2015; 1.

72. National Institutes of Health. National Institutes of Health State of the Science Conference statement on Manifestations and Management of Chronic Insomnia in Adults, 13-15 June 2005. Sleep. 2005;28:1049.

73. Fields WC. "The best cure for insomnia is to get a lot of sleep." Accessed on 20 May 2018. Available from: http://www.doublequotes.net/w-c-fields-quotes/ about-sleep

74. Babson KA, Sottile J, Morabito D. Cannabis, Cannabinoids, and Sleep: A Review of the Literature. Curr Psychiatry Rep. 2017;19(4):23. 